\title{
TRUNCATION ERROR BOUNDS FOR $\pi$-FRACTIONS
}

\author{
BY W. B. GRAGG ${ }^{1}$
}

Communicated by Eugene Isaacson, April 13, 1970

1. Preliminaries. The purpose of this note is to state extensions of the results given in [2] for $g$-fractions. These extensions will be useful for a unification of the theory of inclusion regions for continued fractions associated with certain Hilbert transforms

$$
f(z)=\int_{-\infty}^{+\infty} \frac{d \sigma(t)}{z-t} .
$$

For related results see [1], [3], and [4].

For $-\infty<a<b<+\infty$ let $W(a, b)$ denote the class of nonrational real analytic functions $f(z)$ which are holomorphic for $z \in \operatorname{comp}[a, b]$ and which satisfy $\operatorname{Re}\left[((z-a)(z-b))^{1 / 2} f(z)\right]>0$ in this domain. The principal branch of the square root is assumed.

THEOREM 1. The following alternative characterizations of the class $W(a, b)$ are valid:

(a) $f \in W(a, b)$ if and only if there is a bounded nondecreasing function $\sigma$, with infinitely many points of increase, such that

$$
f(z)=\int_{a}^{b} \frac{d \sigma(t)}{z-t}, \quad z \in \operatorname{comp}[a, b] ;
$$

(b) $f \in W(a, b)$ if and only if $f$ has a (unique) $\pi$-fraction expansion

$$
\begin{aligned}
f(z)= & \frac{\pi_{0}}{\mid z-b}+\frac{b-a}{\mid \frac{1}{z-b}}+\left|\frac{\pi_{1}(z-a)}{z-b}\right|+\frac{b-a}{\mid z-b} \\
& +\mid \frac{\pi_{2}(z-a) \mid}{z-b}+\cdots, \quad z \in \operatorname{comp}[a, b],
\end{aligned}
$$

with $\pi_{n}>0, n \geqq 0$.

2. Inclusion regions. The first inclusion theorem is a consequence of Theorem 1(a).

THEOREM 2. If $f \in W(a, b)$ and $z$ is nonreal then $f(z)$ is contained in the open convex sector $K_{-1}(z)$ bounded by the rays

AMS 1969 subject classifications. Primary 40XX, 12XX.

Key words and phrases. Continued fractions, Stieltjes summation, classical moment problems, orthogonal polynomials.

1 Research sponsored by National Science Foundation Grant GP-9304. 


$$
\stackrel{b}{\kappa_{-1}(z)}: w=\pi /(z-b), \quad \stackrel{a}{\kappa_{-1}}(z): w=\pi /(z-a), \quad 0 \leqq \pi \leqq+\infty .
$$

$K_{-1}(z)$ is precisely the set of all first approximants

$$
w_{1}^{b}(z)=\frac{\pi^{\prime}}{\mid z-b} \mid+\frac{b-a}{\mid 1}+\frac{\pi^{*}(z-a)}{\mid z-b} \quad\left(\pi^{\prime}>0, \pi^{*}>0\right),
$$

or

$$
w_{1}^{a}(z)=\frac{\hat{\pi}}{\mid z-b}\left|+\frac{b-a}{\mid}\right|+\frac{\tilde{\pi}(z-a)}{\mid z-b}+\mid \frac{b-a \mid}{1} \quad(\hat{\pi}>0, \tilde{\pi}>0),
$$

of $\pi$-fractions (1).

This result can now be extended to provide inclusion regions $K_{n}(z)$ which contain $f(z)$, and which are best possible if the first $n+1$ coefficients $\pi_{0}, \pi_{1}, \ldots, \pi_{n}$ are known. For $z$ nonreal the linear fractional transformations

$$
t_{n}(w) \equiv \frac{\pi_{n}}{\mid z-b}+\frac{b-a}{\sqrt{1+(z-a) w} \mid} \quad(n \geqq 0)
$$

are nonsingular with determinants $\pi_{n}(b-a)(z-a)$. Let the composed transformations

$$
T_{n}(w) \equiv t_{0} \circ t_{1} \circ \cdots \circ t_{n}(w) \quad\left(T_{-1}(w) \equiv w\right),
$$

and define

$$
K_{n}(z)=T_{n}\left(K_{-1}(z)\right) \quad(n \geqq-1) .
$$

The transformations $T_{n}$ are also nonsingular linear fractional transformations. From Theorem $2, K_{n}(z)$ is the intersection of two circular disks. Moreover the geometry of the sets $K_{n}(z)$ may be described completely in terms of the approximants

$$
w_{0}^{b}(z), w_{0}^{a}(z), w_{1}^{b}(z), w_{1}^{a}(z), w_{2}^{b}(z), \cdots
$$

of the $\pi$-fraction (1).

Theorem 3. Let $z$ be nonreal, and let $f \in W(a, b)$ have the $\pi$-fraction expansion (1) with approximants (2) and associated sets $K_{n}(z)(n \geqq-1$, $\left.w_{-1}^{b}(z) \equiv \infty, w_{-1}^{a}(z) \equiv 0\right)$. Then the following statements are true for $n \geqq 0$.

(a) $f(z) \in K_{n}(z)$.

(b) $K_{n}(z)$ is precisely the set of all $(n+2)$ th approximants $w_{n+2}^{b}(z)$, $w_{n+2}^{a}(z)$ of $\pi$-fractions (1) with $\pi_{0}, \pi_{1}, \ldots, \pi_{n}$ fixed.

(c) $K_{n}(z)$ is open, bounded, and convex with interior angles

$$
\theta \equiv|\arg [(z-b) /(z-a)]| \text {. }
$$


(d) $K_{n}(z) \subset K_{n-1}(z)$.

(e) $K_{n-1}(z)-K_{n}(z)$ consists of two components $L_{n}^{a}(z)$ and $L_{n}^{b}(z)$. $L_{n}^{a}(z)\left(L_{n}^{b}(z)\right)$ is a circular triangle with vertices

$$
w_{n-1}^{a}(z), \quad w_{n}^{b}(z), \quad w_{n}^{a}(z) \quad\left(w_{n-1}^{b}(z), w_{n}^{a}(z), w_{n}^{b}(z)\right),
$$

and respective interior angles $\theta, \alpha=|\arg (z-a)|, \beta=|\arg (b-z)|$.

The following limiting case of Theorem 3 is a consequence of (e).

Corollary. If $z=x>b$ then

$$
w_{0}^{a}(x)<w_{1}^{a}(x)<w_{2}^{a}(x)<\cdots<w_{2}^{b}(x)<w_{1}^{a}(x)<w_{0}^{b}(x),
$$

and if $z=x<a$ then

$$
w_{0}^{a}(x)<w_{1}^{b}(x)<w_{2}^{a}(x)<\cdots<w_{2}^{b}(x)<w_{1}^{a}(x)<w_{0}^{b}(x) .
$$

3. A priori bounds. The theory of continued fractions, the special form of (1), and the inequality between the arithmetic and geometric means now provide bounds for $w_{n}^{b}(z)-w_{n}^{a}(z)$, and hence also for the diameter of $K_{n}(z)$. Furthermore special examples show that the rate of convergence implied by these bounds is best possible over the class $W(a, b)$.

LEMma. The function

$$
\begin{aligned}
\rho(z) & =\frac{(z-a)^{1 / 2}-(z-b)^{1 / 2}}{(z-a)^{1 / 2}+(z-b)^{1 / 2}} \equiv \frac{1-\left(\frac{z-b}{z-a}\right)^{1 / 2}}{1+\left(\frac{z-b}{z-a}\right)^{1 / 2}} \\
& \equiv \frac{(z-a)-2((z-a)(z-b))^{1 / 2}+(z-b)}{b-a}
\end{aligned}
$$

maps the domain comp $[a, b]$ conformally onto the open unit disk: $|\rho(z)|<1$ for $z \in \operatorname{comp}[a, b]$.

Theorem 4. For $z \in \operatorname{comp}[a, b]$ the diameter of $K_{n}(z)$ satisfies the inequality

$$
\operatorname{diam} K_{n}(z) \leqq \frac{\pi_{0}|\rho(z)|^{n}}{|z-a||z-b| \kappa(\theta)} \quad(n \geqq 0)
$$


with

$$
\kappa(\theta) \equiv \cos \frac{\theta}{2}\left\{\begin{array}{l}
1, \quad 0 \leqq \theta \leqq \frac{\pi}{2}, \\
\sin \theta, \frac{\pi}{2} \leqq \theta<\pi,
\end{array} \quad \theta \equiv\left|\arg \frac{z-b}{z-a}\right|\right.
$$

Moreover

$$
\begin{gathered}
\sup _{s \in W(a, b)} \limsup _{n \rightarrow \infty}\left[\operatorname{diam} K_{n}(z)\right]^{1 / n}=|\rho(z)| . \\
\text { REFERENCES }
\end{gathered}
$$

1. N. I. Ahiezer, The classical moment problem and some related questions in analysis, Fizmatgiz, Moscow, 1961; English transl., Hafner, New York, 1965. MR 27 \#4028; MR 32 \#1518.

2. W. B. Gragg, Truncation error bounds for g-fractions, Numer. Math. 11 (1968), 370-379. MR 37 \#3742.

3. P. Henrici and P. Pfluger, Truncation error estimates for Stieltjes fractions, Numer. Math. 9 (1966), 120-138. MR 35 \#3856.

4. H. S. Wall, Analytic theory of continued fractions, Van Nostrand, Princeton, N. J., 1948. MR 10, 32.

University of California at San Diego, la Jolla, California 92037 